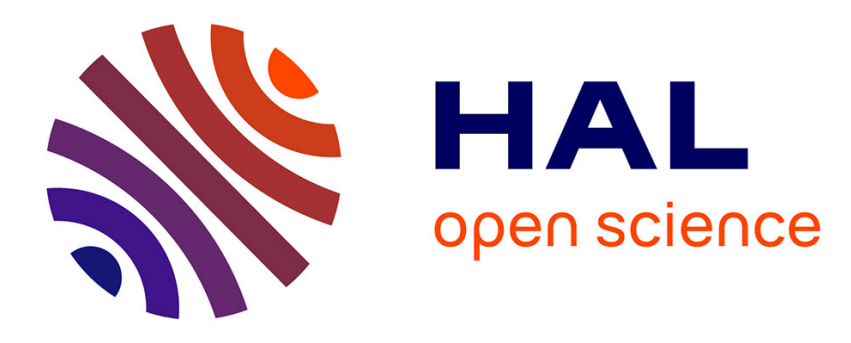

\title{
Problèmes posés par le renouvellement des mélézeins communaux de l'inspection de Gup
}

\author{
A. Py
}

\section{To cite this version:}

A. Py. Problèmes posés par le renouvellement des mélézeins communaux de l'inspection de Gup. Revue forestière française, 1965, 8-9, pp.579-587. 10.4267/2042/24760 . hal-03390003

\section{HAL Id: hal-03390003 https://hal.science/hal-03390003}

Submitted on 21 Oct 2021

HAL is a multi-disciplinary open access archive for the deposit and dissemination of scientific research documents, whether they are published or not. The documents may come from teaching and research institutions in France or abroad, or from public or private research centers.
L'archive ouverte pluridisciplinaire HAL, est destinée au dépôt et à la diffusion de documents scientifiques de niveau recherche, publiés ou non, émanant des établissements d'enseignement et de recherche français ou étrangers, des laboratoires publics ou privés. 
REVUE FORESTIÈRE FRANÇAISE

\section{PROBLĖMES POSES \\ PAR LE RENOUVELLEMENT \\ DES MEELEEZEINS COMMUNAUX \\ DE L'INSPECTION DE GAP}

PAR

A. PY

Ingénieur Principal des Eaux et Forêts

\section{I. - Etat actuel des mélézeins de l'Inspection de Gap}

L'Inspection de Gap comprend:

- 1600 ha de mélézeins aménageables communaux, équiennes en général et âgés de 140 et 200 ans et plus.

- 1400 ha de mélézeins aménageables domaniaux d'origine artificielle âgés de 60 à 90 ans.

Les mélézeins communaux sont traités généralement en futaie régulière avec période d'attente, la régénération étant reculée à une date problématique. Certains massifs sont traités en futaie jardinée mais ce jardinage se réduit à la cueillette des plus gros bois ou des dépérissants. Ces mélézeins sont pâturés le plus souvent 'ovins et bovins). Leur durée de survie étant limitée, il y a lieu de prendre d'urgence les mesures qu'impose leur renouvellement.

Les massifs domaniaux sont en cours d'aménagement. Leur régénération n'est pas à envisager dans l'immédiat. De plus le mélèze ne doit être considéré souvent que comme une essence transitoire de premier boisement. Nous n'en parlerons pas dans cette étude.

Nous ajoutons que l'objet de cette dernière n'est pas d'ordre écologique ni phytosociologique, mais économique, social et financier: il s'agit, compte tenu, bien évidemment, des contraintes que nous imposent la nature et les hommes, de définir une politique raisonnable à partir d'une situation de fait tout aussi contraignante: en matière de mélèze, comme en matière de chêne, de hêtre, de sapin ou d'épicéa, c'est une telle politique qui doit avoir le dernier mot, tel est du moins, à notre avis, ce que recouvre le terme " aménagement ). 


\section{II. - Divers aspects écologiques des mélézeins communaux}

Il faut distinguer généralement dans une même forêt (c'est le cas des forêts d'Orcières, Saint-Léger-les-Mélèzes, Avancon, Ia Batie-Neuve...) :

\section{Mélèzeins. Financement des plantation;}

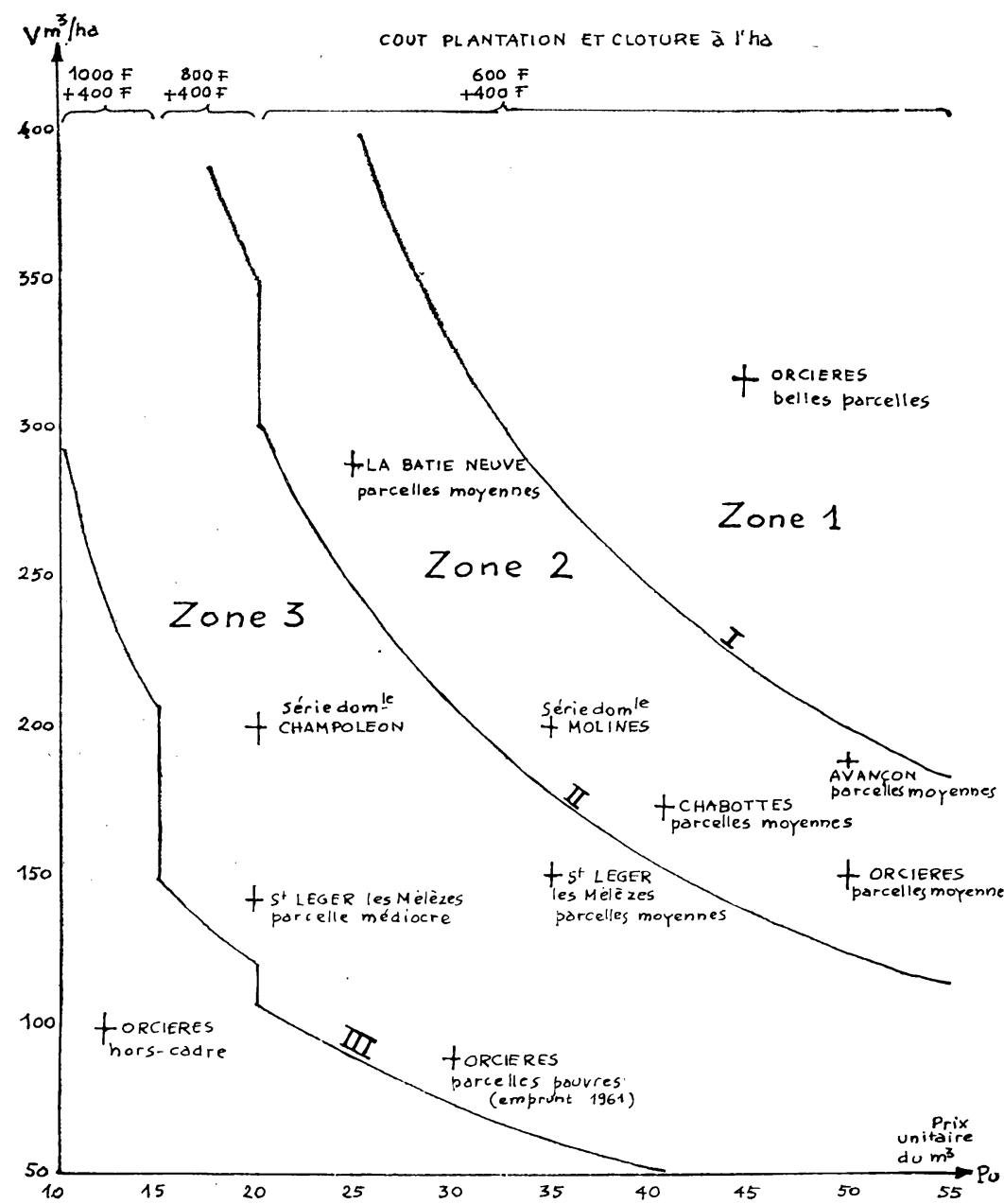

- Les parties basses à pente très rapide, non pâturées de ce fait, situées le plus souvent dans la station du sapin, et dans lesquelles ce dernier s'installe lentement. 
- Les parties hautes, à pente assez rapide ou moyenne, situées en dessous de $1800 \mathrm{~m}$ et pâturées. Pour les municipalités le pâturage y a autant d'importance que la production de bois.

Ce sont des pâturages riches (mélézein à gazon).

- Les parties supérieures à $1800 \mathrm{~m}$ ou exposées aux avalanches portant des peuplements de protection traités en Hors Cadre.

\section{III. - Utilité économique et sociale des mélézeins}

1 - Les mélézeins ont des productions faibles ( 1 à $3 \mathrm{~m}^{3}$ par hectare et par an) et les bois sont commercialisés à bas prix (10 à $40 \mathrm{~F} \mathrm{le} \mathrm{m}^{3}$ sur pied).

Mais il faut remarquer:

- que ces prix sont anormaux: la vogue des sports d'hiver et l'attrait des chalets de montagne peuvent renverser la tendance,

- que, s'agissant de forêts communales considérées souvent comme des forêts de subsistance, les habitants y trouvent souvent des produits auxquels ils sont habitués et qui sont absolument nécessaires à leurs besoins.

2 - Les mélézeins constituent d'excellents terrains de parcours encore largement utilisés.

3 - Pour quelques forêts, le maintien d'un état boisé est imposé par des notions de protection. Mais d'une part cette considération n'est guère valable que pour les forêts domaniales; et d'autre part une hantise exagérée de l'érosion a trop souvent entraîné un excès de prudence dans la conduite des peuplements, le jardinage pied par pied qui, paraît-il, s'imposait, se réduisant à un traitement par coupes d'éclaircie ( légères ) indéfiniment répétées, sans souci des problèmes posés par le vieillissement des massifs.

\section{IV. - Régénération naturelle du mélèze}

Les différentes études faites sur ce sujet montrent la complexité des facteurs qui interviennent.

Une enquête portant sur l'ensemble des mélézeins communaux et domaniaux de l'Inspection a permis de dénombrer trois hectares de semis naturels répartis dans une vingtaine de stations (compte non tenu des lits de torrent, cônes de déjection et couloirs d'avalanches). 
Cependant se présentent toutes les possibilités dans les différents massifs visités quant à :

- la densité des peuplements, leúr ấge, le mélange d'essences ou le contact d'essences diverses, le sol, l'enherbement, l'exercice du pâturage, les microclimats et expositions.

Assurément, depuis trente ans, aucune mesure n'a été prise systématiquement pour obtenir cette régénération et on ne peut conclure à un échec.

Les échos les plus favorables venant du Briançonnais permettent de croire qu'après vingt ans ou plus, dans certains cantons à forte pente, fermés au pâturage, des semis magnifiques s'installent par places. D'autres plus lointains nous parlent d'essences secondaires à introduire.

Les expériences faites par nous ces dernièrès années ne sont pas concluantes: comptages de semis sur potets de replantation dans des parcelles fermées au pâturage, travaux du sol et labours. Le semis lorsqu'il se présente souffre de la concurrence de l'herbe d'autant plus vigoureuse que le pâturage est supprimé et disparaît.

Et pourtant on peut lire dans les vieux procès-verbaux d'aménagement de magnifiques descriptions de fourrés de mélèze.

En 1855, M. Broillard aménageant la forêt de Puy-Saint-Pierre dans le Briançonnais écrivait: " Un fait certain, c'est que dans " le Briançonnais les peuplements de mélèze arrivés à maturité " peuvent avec facilité se régénérer naturellement par la semence. " Le sol de tous les cantons exposés au Nord et à l'Est et dont " le peuplement est clairiéré se recouvre de semis naturels ".

Tout cela bute, se croise. La tentation est de chercher encore si avec les simples on peut guérir le malade. L'état actuel des mélézeins, et notamment leur vieillissement, interdit à notre avis d'attendre le bon plaisir de la nature.

\section{V. - Replantation}

Il faut obtenir l'accord de la commune en cas de fermeture du pâturage dans certains cantons et s'entendre avec elle sur le meilleur emplacement des parcelles à replanter.

Il faut aussi pouvoir financer les travaux. Nous étudierons ce point plus loin.

$\mathrm{Du}$ point de vue technique nous conseillons sur pente forte l'emploi de la tarière mécanique à outil en cœur, précédé si nécessaire du décapage du gazon à la pioche.

L'outil en cœur a l'avantage de laisser la terre en place (La tarière hélicoïdale par contre extrait la terre et lisse les parois du trou). La terre est finement travaillée et de forts plants sont mis en place sans difficulté. Ce " sous-solage ponctuel » semble préférable au travail par potet. Le prix de revient est comparable. 
Sur pente inférieure à $30 \%$, il serait souhaitable d'utiliser un engin automoteur. A ce sujet, notre expérience est la suivante: un engin à flèche porte-tarière fixe est assez peu rentable. Le tracteur doit en effet être mis en place avant l'ouverture de chaque trou. Cette manœuvre est assez longue en raison de l'ensouchement et 'des accidents de terrain.

La solution est l'engin automoteur à flèche orientable et de portée variable. Nous expérimentons actuellement la pelle-grue KRABA, tous terrains, à 4 roues motrices et directrices, équipée d'une tarière hydraulique. La flèche est orientable dans toutes les directions, sa portée varie de $1,50 \mathrm{~m}$ à $3,50 \mathrm{~m}$. Sans se déplacer l'engin peut ouvrir 10 trous.

Le rendement à l'heure atteint 400 trous pour une dépense de $30 \mathrm{~F}$, chauffeur compris.

Un accessoire facile à imaginer permettrait de faire le décapage du gazon en même temps que le travail à la tarière.

La mécanisation doit permettre ainsi de résoudre le problème de la main-d'œuvre en montagne, souvent majeur.

Une dépense importante enfin concerne l'engrillagement qui est absolument nécessaire dans les cantons pâturés.

\section{VI. - Un choix à décider}

\section{- Substitution d'essences.}

Nous sommes d'accord avec de nombreux auteurs sur la nécessité de réduire, partout où c'est possible, la surface des mélézeins purs, par la protection ou l'extension artificielle d'essences plus maniables et de bonne valeur (sapin- épicéa, pin cembro, pin noir, pin sylvestre).

Il est notamment tout indiqué de favoriser l'installation du sapin pectiné, ou de l'introduire (dans son aire) lorsque les massifs ne sont pas pâturés; si en outre ils sont situés sur des versants à forte pente, les futures sapinières pourront être traitées en futaie jardinée, mode de traitement recommandable dans ce cas.

\section{- Station du mélèze.}

Pour notre part nous choisissons la replantation, immédiatement.

Il y a urgence:

1) Les peuplements sont équiennes. Plus ils vieilliront plus difficile sera l'élaboration d'un aménagement.

2) $\mathrm{Si}$ on recule la régénération, le peuplement se clairière, le pâturage prend le pas sur la forêt et le forestier perd pied. 
3) Avec le temps, le capital sur pied diminue, ainsi que les charges et les possibilités d'autofinancement. Les sujétions financières peuvent devenir telles que la forêt est condamnée à se régénérer elle-même ou à disparaitre.

4) Il faut boucher le plus tôt possible le trou de la production à venir du fait de la vieillesse actuelle des peuplements.

5) Il faut utiliser les moyens actuels en personnel, étaler la difficulté, prendre sa part.

\section{VII. - Aménagement en vue de la replantation} lèze.

Nous ne considérons ici que les parcelles à maintenir en mé-

Afin de se mettre dans les conditions de rentabilité les meilleures, ce qui est essentiel en montagne, le traitement en futaie régulière s'impose. La méthode de l'affectation unique est justifiée par l'échéance certaine des années de plantation. $\mathrm{La}$ surface de cette affectation sera plus ou moins grande selon que le relief impose de travailler par bouquets plutôt que par grandes coupes rases. Cette méthode oblige la collectivité propriétaire et le service gestionnaire à proportionner les travaux de régénération à la densité des exploitations et des revenus.

Généralement le relief est facile. Quelle comparaison est possible entre un sol enherbé, ensouché et un sol raviné, surpâturé? Il ne faut pas exagérer le danger de l'érosion.

$\mathrm{Si}$ cela est possible, on travaillera par coupes rases, de un ou plusieurs hectares, au besoin par des exploitations biennales ou triennales; on ouvrira des routes afin de revaloriser les produits et diminuer les frais de plantation et de clôture.

On peut même envisager de soumettre l'affectation à régénérer à une possibilité par contenance particulièrement adaptée à la nécessité d'une programmation rationnelle des travaux de repeuplement, ce qui n'empêche pas la fixation, après inventaire de cette affectation, d'une possibilité indicative par volume.

\section{VIII. - Financement des plantations}

\section{A) Notion de rentabilité immédiate d'une plantation.}

Nous tenons beaucoup à cette notion que nous voudrions développer.

Premier exemple. - Soit une forêt de pin noir d'Auriche âgée de 60 à 80 ans traitée par la méthode de l'affectation unique à la révolution de 80 ans. Surface totale: 800 ha - Période: 20 ans - Volume à l'hectare dans l'affectation: $200 \mathrm{~m}^{3}$. 
La possibilité annuelle est environ: $2200 \mathrm{~m}^{3}$.

Soit un canton distinct de 200 ha récemment planté en pin noir (3 à 5 ans) et dans lequel la réussite des plantations est assurée.

Par un simple jeu d'écriture nous pouvons regrouper ce canton avec la forêt ci-dessus.

La nouvelle possibilité sera augmentée dans la proportion de 8 à 10 , du fait que la surface de l'affectation unique sera augmentée dans cette même proportion, et sera donc égale à : $2750 \mathrm{~m}^{3}$, soit $550 \mathrm{~m}^{3}$ de plus.

La rentabilité immédiate de la plantation est le quotient de la production supplémentaire annuelle par le prix de revient de la plantation. Elle est égale dans le cas présent à $15 \%$.

A elle s'ajoute la rentabilité classique à lointaine échéance de l'ordre de 2 à $3 \%$.

Ainsi ont été adjoints au mélézein communal de Chabottes des communaux aui ont été plantés en pin noir de manière à hâter immédiatement la réalisation du mélézein et à boucher le trou de la production dans 60 ans.

Deuxième exemple. - Supposons que dans un mélézein déterminé l'autofinancement de la replantation soit impossible, la régénération est alors reculée, c'est une nouvelle période d'attente avec éclaircies timides, une cueillette de bois disséminés sans valeur commerciale, l'évolution vers le pré-bois.

Si le reboisement peut être financé par un emprunt, c'est la mise immédiate sur le marché d'un cube supérieur, un revenu argent augmenté pour la commune.

Admettons que ce cube soit égal dans les parcelles à replanter au tiers $d u$ volume à l'hectare. Ce volume ne serait exploité, dans le cas de non plantation, que dans 60 à 80 ans et sous la forme de bois disséminés et sa valeur actuelle correspondante est pratiquement nulle.

Afin de juger si l'emprunt est rentable, il suffit d'appliquer le calcul classique au nouveau montant obtenu en retranchant au montant de l'emprunt le montant des produits supplémentaires exploités du fait de la plantation.

B) Divers modes de financement de la replantation d'un mélézein.

Nous considérons les modes de financement suivants:

- autofinancement avec l'aide de charges $(10 \%)$ et subvention en nature correspondante,

- autofinancement avec l'aide de charges (10\%), subvention en nature correspondant à ces dernières et emprunt. 
Soit V le volume bois d'œuvre à l'hectare des cantons à réaliser, $\mathrm{Pu}$ le prix du mètre cube bois d'œuvre, $\mathrm{p}$ le coût de la plantation à l'hectare, clôture comprise.

Ce coût de plantation augmente d'ailleurs généralement lorsque $\mathrm{Pu}$ diminue.

\section{a) Autofinancement complet ou avec l'aide d'une subvention na- ture. \\ Les forêts pouvant être replantées grâce aux charges ou aux charges + subvention nature sont très faciles à déterminer. Le rapport $\mathrm{V} . \mathrm{Pu} / \mathrm{p}$ doit être supérieur dans les deux cas à un chiffre qui détermine un seuil.}

b) Autofinancement et emprunt.

Intervient alors la rentabilité immédiate des travaux.

Soit une parcelle médiocre de la forêt communale de Saint-Légerles-Mélèzes :

$$
\mathrm{V}=140 \mathrm{~m}^{3} \quad \mathrm{Pu}=120 \mathrm{~F} \quad \mathrm{p}=1200 \mathrm{~F}
$$

La replantation sera financée à l'hectare comme suit:

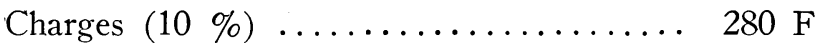

Subvention en nature correspondante ..... $130 \mathrm{~F}$

Emprunt ................... $790 \mathrm{~F}$

Total: $1200 \mathrm{~F}$

Le cube supplémentaire vendu du fait de l'octroi de l'emprunt soit $42 \mathrm{~m}^{3}$ a une valeur de $840 \mathrm{~F}$. Cette somme est supérieure au montant de l'emprunt. Ce dernier a une rentabilité immédiate totale.

c) Représentation graphique.

A titre d'exemple nous avons représenté graphiquement divers cantons de mélézeins de l'Inspection.

Nous avons admis les chiffres suivants: clus):

Coût de la plantation à l'hectare (Clôture comprise, plants ex-

$600 \mathrm{~F}$ pour prix du mètre cube supérieur à $20 \mathrm{~F}$.

$800 \mathrm{~F}$ pour prix du mètre cube compris entre 15 et $20 \mathrm{~F}$.

$1000 \mathrm{~F}$ pour prix du mètre cube inférieur à $15 \mathrm{~F}$.

Fourniture des plants à l'hectare: $400 \mathrm{~F}$.

Chaque forêt est représentée par un point de coordonnées $V$ et $\mathrm{Pu}$. 
Les 3 courbes séparatives indiquent les seuils correspondant aux différents modes de financement.

Courbe I: seuil d'autofinancement par charges (10\%)

Courbe II : seuil de financement par charges $(10 \%)+$ subvention en nature.

Courbe III : seuil de rentabilité immédiate totale de l'emprunt + charges $(10 \%)+$ subvention en nature correspondant à ces dernières.

d) Observations.

10 Classement des parcelles

1) La plupart des parcelles communales de l'Inspection sont assez riches en matériel pour que la replantation soit possible grâce à l'autofinancement par charges de $10 \%$ complété par subvention en nature.

Quelques belles parcelles peuvent être replantées sans aucune subvention.

2) Les parcelles actuelles les plus médiocres des séries de futaie sont susceptibles d'être plantées par emprunt, avec rentabilité immédiate totale.

\section{Préalable à l'aménagement}

Les peuplements des zones 1 et 2 peuvent être aménagés sans aucun préalable.

Si l'on retient la notion de rentabilité immédiate, ce préalable disparait aussi pour les peuplements de la zone 3 qui englobe les forêts ou parcelles pauvres, vouées à disparition dans le cas de non plantation. 